\title{
Statistics of Current Fluctuations and Electron-Electron Interactions in Mesoscopic Coherent Conductors
}

\author{
Dmitri S. Golubev ${ }^{a, c}$, Artem V. Galaktionov ${ }^{b, c}$, and Andrei D. Zaikin ${ }^{b, c}$ \\ ${ }^{a}$ Institut für Theoretische Festkörperphysik, Universität Karlsruhe, 76128 Karlsruhe, \\ Germany; \\ ${ }^{b}$ Forschungszentrum Karlsruhe, Institut für Nanotechnologie, 76021, Karlsruhe, Germany; \\ ${ }^{c}$ P.N. Lebedev Physics Institute, 119991 Moscow, Russia
}

\begin{abstract}
We formulate a general path integral approach which describes statistics of current fluctuations in mesoscopic coherent conductors at arbitrary frequencies and in the presence of interactions. Applying this approach to the non-interacting case, we analyze the frequency dispersion of the third cumulant of the current operator $\mathcal{S}_{3}$ at frequencies well below both the inverse charge relaxation time and the inverse electron dwell time. This dispersion turns out to be important in the frequency range comparable to applied voltages. For comparatively transparent conductors it may lead to the sign change of $\mathcal{S}_{3}$. We also analyze the behavior of the second cumulant of the current operator $\mathcal{S}_{2}$ (current noise) in the presence of electronelectron interactions. In a wide range of parameters we obtain explicit universal dependencies of $\mathcal{S}_{2}$ on temperature, voltage and frequency. We demonstrate that Coulomb interaction decreases the Nyquist noise. In this case the interaction correction to the noise spectrum is governed by the combination $\sum_{n} T_{n}\left(T_{n}-1\right)$, where $T_{n}$ is the transmission of the $n$-th conducting mode. The effect of electron-electron interactions on the shot noise is more complicated. At sufficiently large voltages we recover two different interaction corrections entering with opposite signs. The net result is proportional to $\sum_{n} T_{n}\left(T_{n}-1\right)\left(1-2 T_{n}\right)$, i.e. Coulomb interaction decreases the shot noise at low transmissions and increases it at high transmissions.
\end{abstract}

Keywords: noise, coherence, scattering, interactions, fluctuations

\section{INTRODUCTION}

Experimental and theoretical studies of mesoscopic conductors reveal a rich variety of low temperature properties and effects caused by interplay between scattering, interactions, quantum coherence and electron charge discreteness. Investigations of the whole scope of these effects are of primary interest because of their fundamental importance as well due to rapidly growing number of their potential applications.

A great deal of information is usually obtained from studying electron transport. Additional information can be extracted from investigations of fluctuation effects. During last years much attention has been devoted to the shot noise ${ }^{1}$ described by the second moment of the current operator. One can also study higher order correlators of the current operator thereby extending the amount of information already obtained from electron transport and shot noise. Recently the first experimental study of the third current cumulant in mesoscopic tunnel junctions was reported. ${ }^{2}$

A theoretical framework which enables one to analyze statistics of charge transfer in mesoscopic conductors was developed in Ref. 3. This theory of full counting statistics (FCS) allows to evaluate any cumulant of the current operator in the absence of interactions and in the zero frequency limit. Under these conditions higher order current cumulants were investigated by a number of authors. ${ }^{4-7}$ In order to include interactions and to analyze frequency dispersion of current fluctuations it is necessary to go beyond the FCS theory and to develop a more general real time path integral technique. ${ }^{8-12}$

The goal of the present paper is to address statistics of current fluctuations at non-zero frequencies and in the presence of electron-electron interactions. In what follows we will develop a general path integral approach which allows to perform a complete analysis of electron-electron interaction effects in mesoscopic coherent conductors described by an arbitrary - though energy independent - scattering matrix. This 
approach also provides a straightforward generalization of the FCS theory ${ }^{3}$ to non-zero frequencies. Of central importance in this context is a general and formally exact expression for the real time effective action of a coherent conductor. This expression will be derived and analyzed in various limiting cases in Section II of the paper. With the aid of our technique in Section III we will investigate the frequency dispersion of the third cumulant of the current operator in mesoscopic coherent conductors. Section IV is devoted to the analysis of the electron-electron interaction correction to the second cumulant of the current operator, i.e. to the current noise. The paper is briefly concluded in Section V.

\section{THE MODEL AND EFFECTIVE ACTION}

\subsection{General analysis}

In our analysis we will use the real time path integral formalism developed for the systems of interacting fermions. ${ }^{13}$ After the standard Hubbard-Stratonovich decoupling of the interaction term in the Hamiltonian one can exactly integrate out fermions and arrive at the effective action $S$ which depends on the fluctuating fields $V_{1,2}(t, \boldsymbol{r})$. Let us define

$$
\mathrm{e}^{i S_{0}}=\operatorname{Tr}\left[\mathcal{T} \mathrm{e}^{-i \int_{0}^{t} d t^{\prime} \boldsymbol{H}_{1}\left(t^{\prime}\right)} \hat{\boldsymbol{\rho}}_{0} \tilde{\mathcal{T}} \mathrm{e}^{i \int_{0}^{t} d t^{\prime} \boldsymbol{H}_{2}\left(t^{\prime}\right)}\right]
$$

with the trace taken over the fermionic variables. Here $\hat{\boldsymbol{\rho}}_{0}$ is the initial $N$-particle density matrix of electrons,

$$
\boldsymbol{H}_{1,2}=\sum_{\sigma} \int d^{3} \boldsymbol{r} \hat{\Psi}_{\sigma}^{\dagger}(\boldsymbol{r}) \hat{H}_{1,2}(t) \hat{\Psi}_{\sigma}(\boldsymbol{r}), \quad \hat{H}_{1,2}(t)=-\frac{\nabla^{2}}{2 m}+U(\boldsymbol{r})-e V_{1,2}(t, \boldsymbol{r}) .
$$

Here $\boldsymbol{H}_{1,2}$ are the effective Hamiltonians on the forward and backward parts of the Keldysh contour, $U(\boldsymbol{r})$ describes the static potential, $\mathcal{T}$ and $\tilde{\mathcal{T}}$ are respectively the forward and backward time ordering operators. Integrating out fermions in Eq. (1), we obtain

$$
i S_{0}=2 \operatorname{Tr} \ln \left[1+\left(\hat{u}_{2}^{-1} \hat{u}_{1}-1\right) \hat{\rho}_{0}\right],
$$

where

$$
\hat{u}_{1,2}(t)=\mathcal{T} \exp \left(-i \int_{0}^{t} d t^{\prime} \hat{H}_{1,2}\left(t^{\prime}\right)\right)
$$

are the evolution operators pertaining to the Hamiltonians (2), $\hat{\rho}_{0}$ stands for the initial single-particle density matrix.

The total action of the problem $S$ also includes the contribution of the fluctuating electromagnetic field outside the scatterer. This contribution reads

$$
\begin{aligned}
i S_{\mathrm{ex}}= & -\frac{i}{e^{2}} \int_{0}^{t} d t_{1} d t_{2} \varphi_{S}^{-}\left(t_{1}\right)\left(\int \frac{d \omega}{2 \pi} \frac{\mathrm{e}^{-i \omega\left(t_{1}-t_{2}\right)}}{Z_{S}(\omega)}\right) \dot{\varphi}_{S}^{+}\left(t_{2}\right) \\
& -\frac{1}{2 e^{2}} \int_{0}^{t} d t_{1} d t_{2} \varphi_{S}^{-}\left(t_{1}\right)\left(\int \frac{d \omega}{2 \pi} \operatorname{Re}\left[\frac{1}{Z_{S}(\omega)}\right] \omega \operatorname{coth} \frac{\omega}{2 T} \mathrm{e}^{-i \omega\left(t_{1}-t_{2}\right)}\right) \varphi_{S}^{-}\left(t_{2}\right) .
\end{aligned}
$$

Here $Z_{S}(\omega)$ is the effective linear impedance of the electromagnetic environment, and $\varphi_{S}$ is the quantum phase variable related to the fluctuating voltage $V_{S}$ across the the external leads by means of the equation $\dot{\varphi}_{S}=e V_{S}$. The superscripts $( \pm)$ just reflect the fact that the phase variable is defined on the Keldysh contour, so that symmetric $\left(\varphi_{S}^{+}\right)$and antisymmetric $\left(\varphi_{S}^{-}\right)$combinations of the phases on two branches of this contour should be introduced.

Although the above expression is valid for arbitrary $Z_{S}(\omega)$, for the sake of definiteness and simplicity below we will choose $1 / Z_{S}(\omega)=-i \omega C+1 / R_{S}$, where $C$ is the barrier capacitance and $R_{S}$ is the resistance of external leads (see Fig. 1). The total action of our system then reads

$$
S=S_{\mathrm{ex}}+S_{0} .
$$




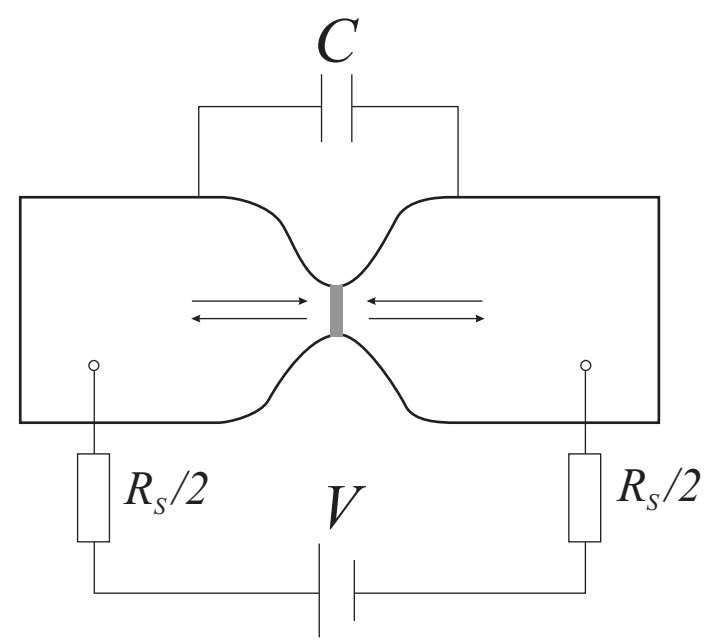

Figure 1. Schematics of the system under consideration. The scatterer is placed in-between two big metallic reservoirs and is characterized by the Landauer conductance $1 / R$ and the effective capacitance $C$. The reservoirs are connected to the voltage source via leads with the total Ohmic resistance $R_{S}$.

In order to evaluate the evolution operators $\hat{u}_{1,2}$ and derive the expression for the action (3) it is necessary to specify the model of a mesoscopic conductor. Here we will adopt the standard model of a (comparatively short) coherent conductor placed in-between two bulk metallic reservoirs, see Fig. 1. The electron dwell time $\tau_{D}$ is supposed to be shorter than any relevant time scale in our problem. Energy and phase relaxation times are, on the contrary, assumed to be long, i.e. inelastic relaxation is allowed in the reservoirs but not inside the conductor. Under these assumptions electron transport through the conductor can be described by the energy independent scattering matrix

$$
\hat{S}=\left(\begin{array}{cc}
\hat{r} & \hat{t}^{\prime} \\
\hat{t} & \hat{r}^{\prime}
\end{array}\right)
$$

and the standard Landauer formula for the conductance $1 / R=\left(e^{2} / \pi\right) \sum_{n} T_{n}=\left(e^{2} / \pi\right) \operatorname{Tr} \hat{t}^{\dagger} \hat{t}$ can be used, where $T_{n}$ defines the transmission for the $n$-th conducting mode.

The effective action (3) can be expressed via the fluctuating phase fields $\varphi_{1,2}$ which are in turn related to the jumps of the fields $V_{1,2}$ across the scatterer as $\dot{\varphi}_{1,2}=e\left(V_{L 1,2}-V_{R 1,2}\right)$, where $V_{L, R}$ are fluctuating in time but constant in space fields in the left and right reservoirs. We note that in this case the right hand side of Eq. (1) differs from the FCS generating functional ${ }^{14}$ only by a gauge transformation.

Within the above model the evolution operators $\hat{u}_{1,2}$ were derived in Refs. 8 and 9. Combining these expressions with Eq. (3) after some algebra we find ${ }^{10}$

$i S_{0}=2 \operatorname{Tr} \ln \left\{\hat{1}+\theta(t-x) \theta(x)\left[\begin{array}{cc}\hat{t}^{\dagger} \hat{t}\left(\mathrm{e}^{i \varphi^{-}(x)}-1\right) & 2 i \hat{t}^{\dagger} \hat{r}^{\prime} \sin \frac{\varphi^{-}(x)}{2} \\ 2 i \hat{r}^{\prime \dagger} \hat{t} \sin \frac{\varphi^{-}(x)}{2} & \hat{t}^{\prime \dagger} \hat{t}^{\prime}\left(\mathrm{e}^{-i \varphi^{-}(x)}-1\right)\end{array}\right]\left[\begin{array}{cc}\rho_{0}(y-x) \mathrm{e}^{i \frac{\varphi^{+}(x)-\varphi^{+}(y)}{2}} & 0 \\ 0 & \rho_{0}(y-x) \mathrm{e}^{i \frac{\varphi^{+}(y)-\varphi^{+}(x)}{2}}\end{array}\right]\right\}$.

Here we introduced $\varphi^{+}=\left(\varphi_{1}+\varphi_{2}\right) / 2, \varphi^{-}=\varphi_{1}-\varphi_{2}$ and

$$
\rho_{0}(x)=\int \frac{d E}{2 \pi} \frac{e^{i E x}}{1+e^{E / T}}=\frac{1}{2} \delta(x)-\frac{i T}{2 \sinh \pi T x} .
$$

Taking the trace in Eq. (7) implies convolution with respect to internal time variables $(x$ and $y)$. The total time span is denoted by $t$. 
Eq. (7) defines a formally exact effective action for a coherent conductor described by an arbitrary energy independent scattering matrix (6). This expression allows to fully determine statistics of current fluctuations at arbitrary frequencies and in the presence of interactions. In the case of equilibrium fluctuations the formula (7) represents a real time analogue of the effective action ${ }^{15-17}$ derived within the Matsubara technique. A formula similar to Eq. (7) was also presented recently in Refs. 11 and 12 . In addition we note that, provided the field $\varphi^{-}$does not depend on time, Eq. (7) coincides with the generating function considered, e.g., in the problem of adiabatic pumping through mesoscopic conductors. ${ }^{18}$

In order to proceed with further calculations it is useful to investigate the properties of the general expression (7) and - whenever it is possible - to find its simplified representations. This goal can be achieved with the aid of several different approximations which can be appropriate in the corresponding physical limits. Some of these approximations and the resulting expressions for the effective action are specified below.

\subsection{Expansion in $T_{n}$}

Provided channel transmissions $T_{n}$ of the scatterer are sufficiently low it can be useful to employ a regular expansion of the action (7) in powers of $T_{n}$. The first order terms of this expansion just yield the standard AES action for tunnel barriers. ${ }^{16}$ Here we will proceed further with this expansion and establish the second order in $T_{n}$ contributions to $S_{0}$. In order to recover all such terms it is necessary to expand the logarithm in Eq. (7) up to the fourth order. After a straightforward - although somewhat tedious - calculation we obtain

$$
\begin{aligned}
i S_{0}= & -\frac{i g}{2 \pi} \int_{0}^{t} d x \dot{\varphi}^{+}(x) \sin \varphi^{-}(x)\left[1+\frac{2(1-\beta)}{3} \sin ^{2} \frac{\varphi^{-}(x)}{2}\right] \\
& -4 g \int_{0}^{t} d x_{1} \int_{0}^{t} d x_{2} \rho_{0}^{2}\left(x_{1}-x_{2}\right) \sin \frac{\varphi^{-}\left(x_{1}\right)}{2} \sin \frac{\varphi^{-}\left(x_{2}\right)}{2} \\
& \times\left\{\beta \cos \left[\varphi^{+}\left(x_{1}\right)-\varphi^{+}\left(x_{2}\right)\right]+(1-\beta) \cos \frac{\varphi^{-}\left(x_{1}\right)-\varphi^{-}\left(x_{2}\right)}{2}\right\} \\
& +\frac{2 i g T^{3}(1-\beta)}{3} \int_{0}^{t} d x_{1} \int_{0}^{t} d x_{2} \int_{0}^{t} d x_{3} \frac{\sin \frac{\varphi^{-}\left(x_{1}\right)}{2} \sin \frac{\varphi^{-}\left(x_{2}\right)}{2} \sin \frac{\varphi^{-}\left(x_{3}\right)}{2}}{\sinh \pi T\left(x_{2}-x_{1}\right) \sinh \pi T\left(x_{3}-x_{2}\right) \sinh \pi T\left(x_{1}-x_{3}\right)} \\
& \times\left\{\sin \left[\varphi^{+}\left(x_{2}\right)-\varphi^{+}\left(x_{1}\right)\right] \cos \frac{\varphi^{-}\left(x_{3}\right)}{2}+\sin \left[\varphi^{+}\left(x_{3}\right)-\varphi^{+}\left(x_{2}\right)\right] \cos \frac{\varphi^{-}\left(x_{1}\right)}{2}\right. \\
& \left.+\sin \left[\varphi^{+}\left(x_{1}\right)-\varphi^{+}\left(x_{3}\right)\right) \cos \frac{\varphi^{-}\left(x_{2}\right)}{2}\right\} \\
& -8 g(1-\beta) \int_{0}^{t} d x_{1} \int_{0}^{t} d x_{2} \int_{0}^{t} d x_{3} \int_{0}^{t} d x_{4} \rho_{0}\left(x_{2}-x_{1}\right) \rho_{0}\left(x_{2}-x_{3}\right) \rho_{0}\left(x_{4}-x_{3}\right) \rho_{0}\left(x_{4}-x_{1}\right) \\
& \times \sin \frac{\varphi^{-}\left(x_{1}\right)}{2} \sin \frac{\varphi^{-}\left(x_{2}\right)}{2} \sin \frac{\varphi^{-}\left(x_{3}\right)}{2} \sin \frac{\varphi^{-}\left(x_{2}\right)}{2} \cos \left[\varphi^{+}\left(x_{1}\right)-\varphi^{+}\left(x_{2}\right)+\varphi^{+}\left(x_{3}\right)-\varphi^{+}\left(x_{4}\right)\right] .
\end{aligned}
$$

Here we introduce the dimensionless conductance of the scatterer $g=2 \sum_{n} T_{n}$ as well as its Fano factor $\beta=\sum_{n} T_{n}\left(1-T_{n}\right) / \sum_{n} T_{n}$. Eq. (9) represents a complete expression for the effective action valid up to the second order in the transmissions $T_{n}$. This expression involves no further approximations and fully accounts for the non-linear dependence on the fluctuating phase fields $\varphi^{ \pm}$.

\subsection{Reflectionless barriers}

Another physically important limit is that of reflectionless barriers $\hat{r}=0$. In this limit the general expression (7) can be reduced to a very simple form by means of the exact procedure which we outline below. The derivation is based on the following property of the Fermi function $n(E)=1 /(1+\exp (E / T))$ :

$$
n(E) n(E+\omega)=\frac{1}{2}\left(1-\operatorname{coth} \frac{\omega}{2 T}\right) n(E)+\frac{1}{2}\left(1+\operatorname{coth} \frac{\omega}{2 T}\right) n(E+\omega) .
$$


This relationship plays the same role as the Ward identity in the Luttinger liquid theory. Rewriting (10) in the coordinate representation one finds

$$
\rho_{0}(x-y) \rho_{0}(y-z)=(f(x-y)+f(y-z)) \rho_{0}(x-z),
$$

where $f(x)=\int \frac{d \omega}{2 \pi} \frac{1}{2}\left(\operatorname{coth} \frac{\omega}{2 T}+1\right) \mathrm{e}^{-i \omega x}=-\frac{i T}{2} \mathcal{P} \operatorname{coth}[\pi T x]+\frac{1}{2} \delta(x)$. Let us formally expand the action (7) in powers of the transmission matrix $\hat{t}^{\dagger} \hat{t}$. This expansion gives rise to the integrals of the type

$$
J_{N}=\int_{0}^{t} d x_{1} d x_{2} \ldots d x_{N} a\left(x_{1}\right) \rho_{0}\left(x_{1}-x_{2}\right) a\left(x_{2}\right) \rho_{0}\left(x_{2}-x_{3}\right) a\left(x_{3}\right) \ldots \rho_{0}\left(x_{N-1}-x_{N}\right) a\left(x_{N}\right) \rho_{0}\left(x_{N}-x_{1}\right),
$$

where $a(x)=\mathrm{e}^{-i \varphi^{-}(x)}-1$. The identity (11) permits to evaluate such integrals exactly. It will be convenient for us to carry out $N-1$ permutations of the variables $x_{j}$ as follows. We start from the set (1) $x_{1}, x_{2}, x_{3} \ldots x_{N}$, and further introduce the following sets of integration parameters

$$
\begin{array}{ccccccc}
(2) & x_{1} & x_{N} & x_{2} & x_{3} & \ldots & x_{N-1} \\
(3) & x_{1} & x_{2} & x_{N} & x_{3} & \ldots & x_{N-1} \\
\ldots & & & & & & \\
N-1) & x_{1} & x_{2} & \ldots & x_{N-2} & x_{N} & x_{N-1},
\end{array}
$$

i.e.. we successively insert $x_{N}$ in-between $x_{1}$ and $x_{2}, x_{2}$ and $x_{3}$, an so on. Symmetrizing the integral (12) with respect to these $N-1$ permutations and excluding $x_{N}$ from the functions $\rho_{0}$ by virtue of the identity (11), we get

$$
\begin{aligned}
J_{N}= & \frac{1}{N-1} \int d x_{1} d x_{2} \ldots d x_{N-1} a\left(x_{1}\right) a\left(x_{2}\right) \ldots a\left(x_{N-1}\right)\left(a\left(x_{1}\right)+a\left(x_{2}\right)+\ldots+a\left(x_{N-1}\right)\right) \\
& \times \rho_{0}\left(x_{1}-x_{2}\right) \rho_{0}\left(x_{2}-x_{3}\right) \ldots \rho_{0}\left(x_{N-1}-x_{1}\right)+\frac{1}{2 \pi(N-1)} \int \frac{d x_{1} d x_{2}}{2 \pi} \frac{\pi^{2} T^{2}}{\sinh ^{2} \pi T\left(x_{1}-x_{2}\right)} a^{N-1}\left(x_{1}\right) a\left(x_{2}\right) .
\end{aligned}
$$

Eq. (14) allows one to relate $J_{N}$ and $J_{N-1}$. After $N-2$ iterations one finds the following result:

$$
J_{N}=\int_{0}^{t} d x \rho_{0}(x, x) a^{N}(x)-\frac{1}{4} \int_{0}^{t} \frac{d x d y}{2 \pi}\left(-\frac{1}{\pi} \frac{\pi^{2} T^{2}}{\sinh ^{2} \pi T(x-y)}\right) \sum_{k=1}^{N-1} \frac{N}{k(N-k)} a^{k}(x) a^{N-k}(y) .
$$

Now we are in a position to evaluate the trace $A=\operatorname{Tr} \ln \left[\hat{1}+\theta(t-x) \theta(x) a(x) \rho_{0}(x-y)\right]$. Exploiting Eq. (15) we obtain

$$
A=\int_{0}^{t} d x \rho_{0}(x, x) \ln (1+a(x))+\frac{1}{8 \pi} \int_{0}^{t} d x d y\left(-\frac{1}{\pi} \frac{\pi^{2} T^{2}}{\sinh ^{2} \pi T(x-y)}\right) \ln (1+a(x)) \ln (1+a(y)) .
$$

Now we recall that in the case of reflectionless barriers $\left(T_{n}=1\right.$ for all $\left.n\right)$ the action (7) reads

$$
i S_{0}=2 N_{\mathrm{ch}}\left\{\operatorname{Tr} \ln \left[\hat{1}+\theta(t-x) \theta(x) a(x) \rho_{R}(y, x)\right]+\operatorname{Tr} \ln \left[\hat{1}+\theta(t-x) \theta(x) a^{*}(x) \rho_{L}(y, x)\right]\right\},
$$

where $N_{\text {ch }}$ is the total number of fully open channels in the conductor and $\rho_{L, R}(y, x)=\mathrm{e}^{i\left[\varphi_{L, R}^{+}(x)-\varphi_{L, R}^{+}(y)\right]} \rho_{0}(y-$ $x)$. Expanding this action in powers of $a(x)$ and $a^{*}(x)$ and applying the formula (16) we observe that the factors $\mathrm{e}^{\varphi_{L, R}^{+}(x)-\varphi_{L, R}^{+}(y)}$ cancel out exactly in all orders except in the first term of Eq. (16). This remaining term should be treated with sufficient care having in mind the formal divergence $\rho_{0}(x, y) \rightarrow \infty$ at $x \rightarrow y$. Finally we obtain

$$
i S_{0}=-\frac{i g}{2 \pi} \int_{0}^{t} d x \dot{\varphi}^{+}(x) W\left(\varphi^{-}(x)\right)+\frac{g}{4} \int_{0}^{t} d x d y \frac{T^{2}}{\sinh ^{2} \pi T(x-y)} W\left(\varphi^{-}(x)\right) W\left(\varphi^{-}(y)\right),
$$

where $g=2 N_{\mathrm{ch}}$ and $W\left(\varphi^{-}\right)$is the $2 \pi$-periodic function equal to $W\left(\varphi^{-}\right)=\varphi^{-}$for $-\pi<\varphi^{-}<\pi$. Note that for the values of $\varphi^{-}$within the latter interval Eq. (18) exactly coincides with the action for an Ohmic conductor. Finally we point out that the existence of the exact solution (18) in the limit $\hat{r}=0$ is not at all surprising since in that limit the problem can also be exactly treated by means of the bosonization technique. ${ }^{19}$ 


\subsection{Local in time part of the action}

The above results for $S_{0}$ demonstrate that the effective action can be split into two parts,

$$
S_{0}=S_{\text {local }}+S_{\text {nonlocal }}
$$

determined respectively by local and non-local in time expressions. One can also observe that the term $S_{\text {local }}$ is linear in $\dot{\varphi}^{+}$. Such splitting can be performed for arbitrary transmission values. It turns out that the local part of the effective action can be found exactly in all orders in $T_{n}$.

In order to derive this exact expression let us first fix $\varphi^{+}=e V t$ and $\varphi^{-}=$const. It is easy to demonstrate $^{10}$ that under these restrictions the action $S_{0}$ reduces to the FCS generating function ${ }^{3}$

$$
i S_{0} \rightarrow-\frac{T t}{\pi} \sum_{n}\left[v^{2}-\operatorname{Arccosh}^{2}\left(T_{n} \cosh \left(v-i \varphi^{-}\right)+\left(1-T_{n}\right) \cosh v\right)\right],
$$

where $\operatorname{Arccosh} x=\ln \left(x+\sqrt{x^{2}-1}\right)$ and $v=e V / 2 T$. The part $S_{\text {local }}$ can be identified from this generating function in the limit $v \ll 1$. Keeping only linear in $v$ terms one can rewrite Eq. (20) in the form

$$
i S_{0} \rightarrow-\frac{i e V t}{\pi} \sum_{n} \frac{T_{n} \sin \varphi^{-}\left(\frac{\pi}{2}-\arctan \frac{1-2 T_{n} \sin ^{2} \frac{\varphi^{-}}{2}}{2 \sin \frac{\left|\varphi^{-}\right|}{2} \sqrt{T_{n}\left(1-T_{n} \sin ^{2} \frac{\varphi^{-}}{2}\right)}}\right)}{2 \sin \frac{\left|\varphi^{-}\right|}{2} \sqrt{T_{n}\left(1-T_{n} \sin ^{2} \frac{\varphi^{-}}{2}\right)}}+\mathcal{O}\left(v^{2}\right) .
$$

Since $S_{\text {local }}$ should be determined by the local in time combination one can now replace $e V$ by an arbitrary function of time $\dot{\varphi}^{+}$and also allow for arbitrary changes of $\varphi^{-}$in time. This observation immediately brings us to the final result

$$
i S_{\text {local }}=-\frac{i}{\pi} \int_{0}^{t} d t^{\prime} \dot{\varphi}^{+} \sum_{n} \frac{T_{n} \sin \varphi^{-}\left(\frac{\pi}{2}-\arctan \frac{1-2 T_{n} \sin ^{2} \frac{\varphi^{-}}{2}}{2 \sin \frac{\left|\varphi^{-}\right|}{2} \sqrt{T_{n}\left(1-T_{n} \sin ^{2} \frac{\varphi^{-}}{2}\right)}}\right)}{2 \sin \frac{\left|\varphi^{-}\right|}{2} \sqrt{T_{n}\left(1-T_{n} \sin ^{2} \frac{\varphi^{-}}{2}\right)}} .
$$

Unfortunately the above exact expression for $S_{\text {local }}$ alone is not sufficient for a full description of quantum properties of our system and no such simple derivation procedure exists for the non-local in time part $S_{\text {nonlocal }}$. Nonetheless, Eq. (22) provides an important piece of information about the properties of the effective action $S_{0}$ and it can be successfully employed in further calculations.

\subsection{Expansion in $\varphi^{-}$}

In a large number of physical situations it is sufficient to restrict the analysis to the limit of large scatterer conductances $g \gg 1$. Either in this limit or, equally, in the case $g_{S}=2 \pi / e^{2} R_{S} \gg 1$, fluctuations of the quantum field $\varphi^{-}$are considerably suppressed almost at all energies/frequencies. In this case it is useful to expand the exact expression for $S_{0}$ in powers of $\varphi^{-}$keeping the full non-linearity in $\varphi^{+}$in all orders of this expansion. Below we will present the result of this expansion up to the third order in $\varphi^{-}$. The corresponding derivation has been worked out in Ref. 9. It yields

$$
\begin{aligned}
i S_{0}= & -\frac{i g}{2 \pi} \int d x\left(\varphi^{-}(x)-\frac{\beta}{6}\left[\varphi^{-}(x)\right]^{3}\right) \dot{\varphi}^{+}(x) \\
& +\frac{g}{4 \pi^{2}} \int d x_{1} d x_{2} \frac{\pi^{2} T^{2}}{\sinh ^{2} \pi T\left(x_{1}-x_{2}\right)} \varphi^{-}\left(x_{1}\right) \varphi^{-}\left(x_{2}\right)\left[1-\beta+\beta \cos \left(\varphi^{+}\left(x_{1}\right)-\varphi^{+}\left(x_{2}\right)\right)\right] \\
& +\frac{\pi i \gamma T^{3}}{6 e^{2} R} \int d x_{1} d x_{2} d x_{3} \frac{\varphi^{-}\left(x_{1}\right) \varphi^{-}\left(x_{2}\right) \varphi^{-}\left(x_{3}\right)}{\sinh \left[\pi T\left(x_{2}-x_{1}\right)\right] \sinh \left[\pi T\left(x_{3}-x_{2}\right)\right] \sinh \left[\pi T\left(x_{1}-x_{3}\right)\right]} \\
& \times\left\{\sin \left[\varphi^{+}\left(x_{2}\right)-\varphi^{+}\left(x_{1}\right)\right]+\sin \left[\varphi^{+}\left(x_{3}\right)-\varphi^{+}\left(x_{2}\right)\right]+\sin \left[\varphi^{+}\left(x_{1}\right)-\varphi^{+}\left(x_{3}\right)\right]\right\}
\end{aligned}
$$


where we have defined $\gamma=\sum T_{n}^{2}\left(1-T_{n}\right) / \sum T_{n}$. Obviously, the local in time part of Eq. (23) can also be recovered by a direct expansion of Eq. (22) in powers of $\varphi^{-}$.

Below we will make use of the result (23) in order to evaluate the frequency dependence of the third cumulant of the current operator and to derive the interaction correction to the shot noise spectrum.

\section{FREQUENCY DEPENDENCE OF THE THIRD CURRENT CUMULANT}

To begin with, let us recall that the standard way to describe the current noise in our system is to evaluate the symmetrized correlation function

$$
\mathcal{S}\left(t, t^{\prime}\right)=\frac{1}{2}\left\langle\hat{I}(t) \hat{I}\left(t^{\prime}\right)+\hat{I}\left(t^{\prime}\right) \hat{I}(t)\right\rangle-\left\langle\hat{I}^{2},\right.
$$

where $\hat{I}$ is the current operator in the circuit of Fig. 1. After the Fourier transformation within our path integral technique the same quantity can be expressed as follows

$$
\mathcal{S}_{2}(\omega)=-e^{2} \int d \tau \mathrm{e}^{i \omega \tau} \int \mathcal{D} \varphi_{1} \mathcal{D} \varphi_{2} \frac{\delta^{2}}{\delta \varphi^{-}(t+\tau) \delta \varphi^{-}(t)} \mathrm{e}^{i S\left[\varphi^{+}, \varphi^{-}\right]}
$$

Here the path integral is taken over all possible configurations of the phases $\varphi^{ \pm}$. In this way the effect of Coulomb interaction on current noise is fully accounted for by Eq. (24). This effect will be studied in the next section.

Proceeding along the same lines one can also define higher cumulants of the current operator. Below we will only analyze the frequency dependence of the third cumulant. In doing so, we will ignore interactions. In this case the path integral is dominated by the trajectories $\varphi^{+}(t)=e V t$ and $\varphi^{-}=0$, the latter condition being imposed after taking the functional derivatives:

$$
\mathcal{S}_{3}\left(\omega_{1}, \omega_{2}\right)=\left.e^{3} \int d \tau_{1} d \tau_{2} \mathrm{e}^{i \omega_{1} \tau_{1}} \mathrm{e}^{i \omega_{2} \tau_{2}} \frac{\delta^{3} S}{\delta \varphi^{-}(t) \delta \varphi^{-}\left(t-\tau_{1}\right) \delta \varphi^{-}\left(t-\tau_{2}\right)}\right|_{\varphi^{-}=0} .
$$

It is important to emphasize that the current through the scatterer should be treated as a quantum operator. Since in general the current operators do not commute if taken at different times, the question arises about the proper ordering of such operators in the third and higher order correlation functions. One can verify that the definition (25) corresponds to the following combination of the correlation functions:

$$
\begin{aligned}
\mathcal{S}_{3}\left(t_{1}, t_{2}, t_{2}\right)= & \frac{1}{8}\left\{\left\langle\hat{I}\left(t_{1}\right)\left(\mathcal{T} \hat{I}\left(t_{2}\right) \hat{I}\left(t_{3}\right)\right)\right\rangle+\left\langle\left(\tilde{\mathcal{T}} \hat{I}\left(t_{2}\right) \hat{I}\left(t_{3}\right)\right) \hat{I}\left(t_{1}\right)\right\rangle+\left\langle\hat{I}\left(t_{2}\right)\left(\mathcal{T} \hat{I}\left(t_{1}\right) \hat{I}\left(t_{3}\right)\right)\right\rangle+\left\langle\left(\tilde{\mathcal{T}} \hat{I}\left(t_{1}\right) \hat{I}\left(t_{3}\right)\right) \hat{I}\left(t_{2}\right)\right\rangle\right. \\
& \left.+\left\langle\hat{I}\left(t_{3}\right)\left(\mathcal{T} \hat{I}\left(t_{1}\right) \hat{I}\left(t_{2}\right)\right)\right\rangle+\left\langle\left(\tilde{\mathcal{T}} \hat{I}\left(t_{1}\right) \hat{I}\left(t_{2}\right)\right) \hat{I}\left(t_{3}\right)\right\rangle+\left\langle\mathcal{T} \hat{I}\left(t_{1}\right) \hat{I}\left(t_{2}\right) \hat{I}\left(t_{3}\right)\right\rangle+\left\langle\tilde{\mathcal{T}} \hat{I}\left(t_{1}\right) \hat{I}\left(t_{2}\right) \hat{I}\left(t_{3}\right)\right\rangle\right\} \\
& -\frac{1}{2}\left\langle\hat{I}\left(t_{1}\right)\right\rangle\left\langle\hat{I}\left(t_{2}\right) \hat{I}\left(t_{3}\right)+\hat{I}\left(t_{3}\right) \hat{I}\left(t_{2}\right)\right\rangle-\frac{1}{2}\left\langle\hat{I}\left(t_{2}\right)\right\rangle\left\langle\hat{I}\left(t_{1}\right) \hat{I}\left(t_{3}\right)+\hat{I}\left(t_{3}\right) \hat{I}\left(t_{1}\right)\right\rangle \\
& -\frac{1}{2}\left\langle\hat{I}\left(t_{3}\right)\right\rangle\left\langle\hat{I}\left(t_{1}\right) \hat{I}\left(t_{2}\right)+\hat{I}\left(t_{2}\right) \hat{I}\left(t_{1}\right)\right\rangle+2\left\langle\hat{I}\left(t_{1}\right)\right\rangle\left\langle\hat{I}\left(t_{2}\right)\right\rangle\left\langle\hat{I}\left(t_{3}\right)\right\rangle .
\end{aligned}
$$

This combination is of interest in the light of possible experimental investigations of current fluctuations. Indeed, the correlation function $\mathcal{S}_{2}$ is important because the symmetric combination of voltages $V^{+}$can be viewed as a classical, measurable, voltage. ${ }^{14}$ The noise is deduced from the measurable product $V^{+}\left(t_{1}\right) V^{+}\left(t_{2}\right)$, which is related to the symmetric correlator $\mathcal{S}_{2}(23)$ or (24). Analogously, the measured product $V^{+}\left(t_{1}\right) V^{+}\left(t_{2}\right) V^{+}\left(t_{3}\right)$ is related to the correlation function of the current operators $\mathcal{S}_{3}$ defined in Eq. (26), see also Refs. 14 and 20 for further discussion of this point.

Let us substitute the above expressions into Eq. (23) and, after taking derivatives over $\varphi^{-}$, set $\varphi^{+}(\tau)=$ $e V \tau$ and $\varphi^{-} \rightarrow 0$. This is sufficient provided the time differences $\left|t_{1}-t_{2}\right|,\left|t_{1}-t_{3}\right|$ exceed the charge relaxation time $\tau_{R C}$ and provided $e V \ll 1 / \tau_{R C}$. Eq. (23) then yields

$$
\mathcal{S}_{3}=\beta e^{2} \bar{I} \delta\left(t_{1}-t_{2}\right) \delta\left(t_{1}-t_{3}\right)-\frac{4 \pi e \gamma}{R} f\left(t_{2}-t_{1}\right) f\left(t_{3}-t_{2}\right) f\left(t_{1}-t_{3}\right),
$$


where $f(\tau)=T \sin (e V \tau / 2) / \sinh (\pi T \tau)$. Performing the Fourier transformation

$$
\mathcal{S}_{3}\left(\omega_{1}, \omega_{2}\right)=\int d \tau_{1} d \tau_{2} \mathrm{e}^{i \omega_{1} \tau_{1}+i \omega_{2} \tau_{2}} \mathcal{S}_{3}\left(t_{1}, t_{1}-\tau_{1}, t_{1}-\tau_{2}\right),
$$

we arrive at the final result

$$
\begin{array}{r}
\mathcal{S}_{3}=\beta e^{2} \bar{I}-2 \gamma e^{2} \bar{I} F\left(v, w_{1}, w_{2}\right), \\
F=\frac{\sinh ^{3}(v / 2)}{4 v} \int_{-\infty}^{\infty} \frac{d \omega}{\chi(\omega) \chi\left(\omega-w_{1}\right) \chi\left(\omega+w_{2}\right)} .
\end{array}
$$

Here we defined $v=e V / T, w_{1,2}=\omega_{1,2} / 2 T$ and

$$
\chi(\omega)=\cosh ^{2} \omega+\sinh ^{2}(v / 4) .
$$

Eqs. (28)-(30) represent the main result of this section. They fully describe the third cumulant of the current operator at voltages and frequencies smaller than both $1 / \tau_{R C}$ and $1 / \tau_{D}$.

Let us briefly analyze Eqs. (28)-(30) in various limits. For $\omega_{1,2}=0$ we recover the well known result ${ }^{5}$

$$
F(v, 0,0)=1+3 \frac{1-(\sinh v / v)}{\cosh v-1},
$$

which in turn yields $F \rightarrow 1$ in the limit of large voltages $v \gg 1$. Eq. (31) also holds for $w_{1,2} \ll v$.

In the limit $v \ll 1$ one finds

$$
\begin{gathered}
F(v \ll 1, w, 0)=F(v \ll 1, w,-w)=\frac{9 \sinh w+\sinh (3 w)-12 w \cosh w}{48 \sinh ^{5} w} v^{2}, \\
F(v \ll 1, w, w)=\frac{\sinh (4 w)+4 \sinh (2 w)-8 w \cosh (2 w)-4 w}{128 \sinh ^{5} w \cosh ^{3} w} v^{2} .
\end{gathered}
$$

These equations demonstrate that at large frequencies $w \gg 1$ the function $F$ decays exponentially with $w$. From Eqs. (32) and (33) we find respectively $F \propto v^{2} e^{-2 w} / 3$ and $F \propto v^{2} e^{-4 w}$.

Finally let us turn to the most interesting limit of low temperatures, in which case one always has $v, w_{1}, w_{2} \gg 1$. Neglecting small corrections $\sim 1 / v, 1 / w$ we obtain

$$
\begin{array}{rll}
F\left(v, w_{1}, w_{2}\right)=1-2\left|\frac{w_{12}}{v}\right|, & \text { if } & 2\left|w_{12}\right|<|v|, \\
F\left(v, w_{1}, w_{2}\right)=0, & \text { if } & 2\left|w_{12}\right|>|v| .
\end{array}
$$

Here the value $w_{12}$ is defined differently depending on the sign of the product $w_{1} w_{2}$. For $w_{1} w_{2}>0$ we have $w_{12}=w_{1}+w_{2}$ while in the opposite case $w_{1} w_{2}<0$ we define $w_{12}=\max \left[\left|w_{1}\right|,\left|w_{2}\right|\right]$. We observe that in both cases the function $F$ depends linearly on frequency and vanishes as soon as $\left|w_{12}\right|$ exceeds $|v| / 2$.

At arbitrary values of $v, w_{1}$ and $w_{2}$ the integral (29) can be evaluated numerically. The corresponding result for the function $F\left(v, w_{1}, w_{2}\right)$ is presented in Fig. 2.

Let us emphasize again that in the course of our analysis of the correlator $\mathcal{S}_{3}\left(\omega_{1}, \omega_{2}\right)$ we have ignored electron-electron interactions and also assumed that the external impedance $R_{S}$ equals to zero, i.e. the voltage source is directly attached to the scatterer. In realistic experimental setups the latter condition may be violated, in which case the third cumulant can be substantially modified ${ }^{21}$ by current fluctuations in the external environment (leads). Within our formalism this effect can be accounted for by the external impedance $Z_{S}(\omega)$. 


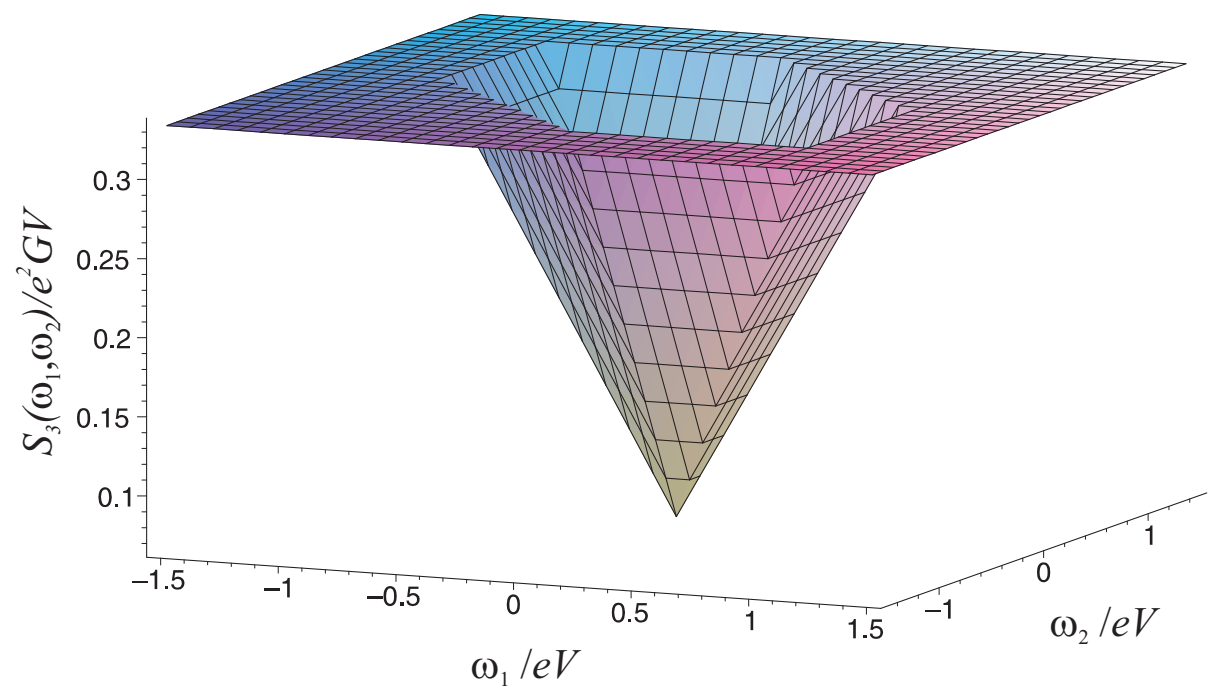

Figure 2. Frequency dependence for the third cumulant of the current operator $\mathcal{S}_{3}\left(\omega_{1}, \omega_{2}\right)$ evaluated by means of Eqs. (28)-(30) at $T=0$ for a short diffusive conductor with $\beta=1 / 3$ and $\gamma=2 / 15$.

\section{CURRENT NOISE IN THE PRESENCE OF INTERACTIONS}

Let us now turn to the second cumulant (23) and explicitly account both for interactions and the external leads $R_{S}$. It is clear that fluctuations both in the scatterer and in the external resistor will affect the correlator (23), i.e. the latter should in general depend on both $R$ and $R_{S}$. Let us - in addition to (23) - introduce another correlator $\tilde{\mathcal{S}}\left(t, t^{\prime}\right)$ defined by the same Eq. (23) in which one should substitute the current operator across the scatterer $\hat{I} \rightarrow \hat{I}_{\mathrm{sc}}$. The two correlators $\mathcal{S}\left(t, t^{\prime}\right)$ and $\tilde{\mathcal{S}}\left(t, t^{\prime}\right)$ are not independent. With the aid of the current conservation condition and performing the Fourier transformation with respect to $t-t^{\prime}$, one easily obtains the relation between these correlation functions:

$$
\tilde{\mathcal{S}}_{\omega}=\frac{R_{S}^{2}}{R_{0}^{2}}\left(1+\omega^{2} R_{0}^{2} C^{2}\right) \mathcal{S}_{\omega}-\frac{R_{S}}{R^{2}}\left(1+\omega^{2} R^{2} C^{2}\right) \omega \operatorname{coth} \frac{\omega}{2 T},
$$

where $R_{0}=R R_{S} /\left(R+R_{S}\right)$. The second term is due the noise produced by the external resistor $R_{S}$ which has to be subtracted in order to arrive at $\tilde{\mathcal{S}}_{\omega}$.

In general also the correlator $\tilde{\mathcal{S}}_{\omega}$ depends on both $R$ and $R_{S}$. However, in the limit $R_{S} \gg R$ the dependence on the shunt resistance is weak and can be neglected. In this case the interaction correction to the current noise spectrum depends only on the properties of the scatterer. Below we will present our results only in this limit. More general expressions can be found in Ref. 9.

As before, we will assume that the condition $g+g_{S} \gg 1$ is fulfilled throughout our calculation. Combining the result (23) with the definition (24) and having in mind the relation (36) one can directly evaluate the correlator $\delta \tilde{\mathcal{S}}_{\omega}$ in the presence of interactions. In the important limit $\omega, e V, T \ll 1 / R C$ one finds

$$
\begin{aligned}
S_{2}(\omega)= & \frac{2(1-\beta)}{R} \omega \operatorname{coth} \frac{\omega}{2 T}+\beta e \tilde{I}\left(\frac{\omega}{e}+V\right) \operatorname{coth} \frac{\omega+e V}{2 T}+\beta e \tilde{I}\left(\frac{\omega}{e}-V\right) \operatorname{coth} \frac{\omega-e V}{2 T} \\
& +\pi \gamma T^{3} e^{2} \int_{0}^{\infty} d x \int_{0}^{\infty} d y \frac{\left(1-\mathrm{e}^{-x / R C}\right)(\cos e V y-\cos e V x) \cos \omega y}{\sinh \pi T x \sinh \pi T y} \\
& \times\left(\frac{1}{\sinh \pi T(x-y)}-\frac{1}{\sinh \pi T(x+y)}\right),
\end{aligned}
$$

where

$$
\tilde{I}(V)=\frac{V}{R}-\frac{e}{\pi} \int_{0}^{\infty} d t \frac{\pi^{2} T^{2}}{\sinh ^{2} \pi T t}\left(1-\mathrm{e}^{-t / R C}\right) \sin e V t
$$


The result (37) covers several important regimes. In the limit of weak tunneling $\beta \rightarrow 1$ and $\gamma \rightarrow 0$ we find

$$
S_{2}(\omega)=e \tilde{I}\left(\frac{\omega}{e}+V\right) \operatorname{coth} \frac{\omega+e V}{2 T}+e \tilde{I}\left(\frac{\omega}{e}-V\right) \operatorname{coth} \frac{\omega-e V}{2 T}
$$

Here the function $I(V)$ is just the $I-V$ curve of a tunnel barrier modified by weak Coulomb blockade corrections. Eq. (39) can be viewed as a generalization of the result. ${ }^{22}$ This formula also agrees with the result ${ }^{23}$ derived in the limit $g \ll 1$.

It is worth pointing out that Eq. (37) can be reduced to the form (39) only in the case of tunnel barriers. Otherwise the interaction correction to the current noise cannot be obtained from the corresponding correction to the $I-V$ curve except for equilibrium situations where the fluctuation-dissipation theorem (FDT) can be employed. In order to illustrate this point and to present our results beyond the tunneling limit let us split the function $\tilde{\mathcal{S}}$ into two parts:

$$
\tilde{\mathcal{S}}\left(t, t^{\prime}\right)=\tilde{\mathcal{S}}^{\mathrm{ni}}\left(t, t^{\prime}\right)+\delta \tilde{\mathcal{S}}\left(t, t^{\prime}\right),
$$

where $\tilde{\mathcal{S}}^{\text {ni }}$ is the noninteracting contribution to the current noise ${ }^{1}$ and $\delta \tilde{\mathcal{S}}$ is the correction due to electronelectron interactions inside the scatterer. Let us define the average voltage across the scatterer $V=$ $V_{x} R /\left(R+R_{S}\right)$ and consider first the limit of relatively small voltages. At sufficiently large temperatures and/or frequencies we find

$$
\begin{array}{cc}
\delta \tilde{\mathcal{S}}_{\omega}=-\frac{2 \beta E_{C}}{3 R}, & \text { if } T \gg g E_{C},|e V|,|\omega|, \\
\delta \tilde{\mathcal{S}}_{\omega}=-\frac{\beta E_{C}}{R}, & \text { if }|\omega| \gg T, g E_{C},|e V| .
\end{array}
$$

At lower temperatures and frequencies we obtain

$$
\begin{gathered}
\delta \tilde{\mathcal{S}}_{\omega}=-\frac{4 \beta T}{R_{q}} \ln \frac{g E_{C}}{T}, \quad \text { if }|\omega|,|e V| \ll T \ll g E_{C}, \\
\delta \tilde{\mathcal{S}}_{\omega}=-\frac{2 \beta|\omega|}{R_{q}} \ln \frac{g E_{C}}{|\omega|}, \quad \text { if } T,|e V| \ll|\omega| \ll g E_{C} .
\end{gathered}
$$

These results apply as long as either temperature or frequency exceeds the parameter $g E_{C} \exp (-g / 2)$.

As we have already pointed out, the above expressions could also be anticipated from FDT combined with the results. ${ }^{8}$ Indeed, in the limit of low voltages the current noise is described by the standard Nyquist formula. Hence, in order to satisfy FDT one should simply substitute the effective conductance ${ }^{8}$ into this formula. In this way one immediately reproduces Eqs. (41) and (43).

Now let us turn to the case of relatively large voltages $V$ where the shot noise becomes important. In this case the correction to the noise power spectrum is found to be proportional to the parameter

$$
\beta-2 \gamma=\frac{\sum_{n} T_{n}\left(1-T_{n}\right)\left(1-2 T_{n}\right)}{\sum_{n} T_{n}}
$$

We obtain

$$
\begin{array}{r}
\delta \tilde{\mathcal{S}}_{\omega}=-\frac{2(\beta-2 \gamma)|e V|}{R_{q}} \ln \frac{g E_{C}}{|e V|}, \quad \text { if } T,|\omega| \ll|e V| \ll g E_{C}, \\
\delta \tilde{S}_{\omega}=-\frac{(\beta-2 \gamma) E_{C}}{R}, \quad \text { if }|e V| \gg T, g E_{C},|\omega| .
\end{array}
$$

We note that this correction can be either negative or positive depending on the relation between the parameters $\beta$ and $\gamma$. Thus, in contrast to the limit of low voltages (Nyquist noise), one cannot conclude that shot noise is always reduced by interactions. This reduction occurs only for conductors with relatively 


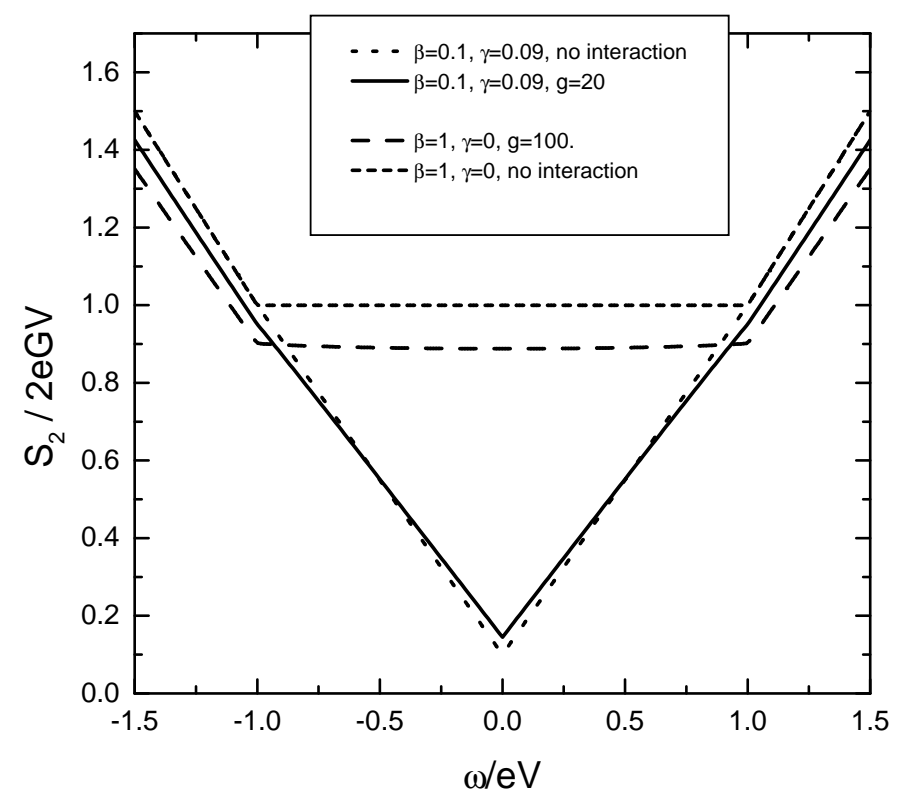

Figure 3. Frequency dependence of the noise correlator $\mathcal{S}_{2}$ at $T=0$ and for various parameters of the system.

low transmissions $\beta>2 \gamma$, while for systems with higher transmissions the net effect of the electron-electron interaction enhances the shot noise. In the important case of diffusive conductors one has $\beta=1 / 3, \gamma=2 / 15$ and, hence, $\beta-2 \gamma=1 / 15$. In this case the shot noise is reduced by interactions.

The above results have a transparent physical interpretation. At low voltages the power spectrum of the Nyquist noise is proportional to the system conductance $\propto \sum_{n} T_{n}$. Since in the presence of interactions the conductance acquires a correction proportional to $\beta$, the interaction correction to the Nyquist noise should scale with the same parameter. On the other hand, shot noise is determined by the combination $\sum_{n}\left(T_{n}-\right.$ $\left.T_{n}^{2}\right)$. Accordingly, the interaction correction to the shot noise power should consist of two contributions. One of them comes from $\sum_{n} T_{n}$ and is again proportional to $\beta$. Another contribution originates from the interaction correction to $\sum_{n} T_{n}^{2}$ which turns out to scale as $2 \gamma$. Since these two corrections enter with opposite signs we immediately arrive at the combination (45).

We also point out that the third cumulant of the current operator for noninteracting electrons is known ${ }^{5}$ to be proportional to the parameters $\beta$ and $\beta-2 \gamma$ respectively at low and high voltages. Following the same arguments as above one can anticipate that the interaction correction to the third cumulant should scale as $\beta-2 \gamma$ at low voltages and as $\beta-6 \gamma+6 \delta$ at high voltages, where $\delta=\sum_{n} T_{n}^{3}\left(1-T_{n}\right) / \sum_{n} T_{n}$ Technically, in order to determine the interaction correction to the third cumulant $\mathcal{S}_{3}$ it is necessary to expand the exact effective action $S_{0}(7)$ up to the fourth order in $\varphi^{-}$. Along with this interaction correction such expansion allows to determine the full frequency dependence of the fourth cumulant $\mathcal{S}_{4}$ in the absence of interactions. This rule also applies to higher cumulants, i.e. the lowest order interaction correction to the $n$-th current cumulant $\mathcal{S}_{n}$ is determined by $\mathcal{S}_{n+1}$ for all values of $n$. This fact can also be proven by means of the renormalization group analysis recently developed in Refs. 11 and 12 .

\section{CONCLUDING REMARKS}

In this paper we have presented a general path integral approach which allows to describe statistics of current fluctuations in mesoscopic coherent conductors at arbitrary frequencies and in the presence of interactions. This approach enables one to establish a complete expression for the effective action of coherent conductors described by an arbitrary - though energy independent - scattering matrix and to elucidate a profound relation between full counting statistics and electron-electron interaction effects in coherent mesoscopic 
conductors. Further extention of our technique to more complicated structures described by the energy dependent scattering matrices is possible and was recently worked out. ${ }^{24}$

Restricting ourselves to the non-interacting case, we have analyzed frequency dispersion of the third cumulant of the current operator. This dispersion was found negligible only in the case of tunnel junctions, while in a general case it turns out to be important in the frequency range comparable to $\mathrm{eV}$. For instance, in the important case of diffusive conductors and at $T=0$ the quantity $\mathcal{S}_{3}$ changes by the factor 5 depending on whether relevant frequencies are below or above $\mathrm{eV}$. For conductors with $\beta<2 \gamma$ even the sign of $\mathcal{S}_{3}$ differs in these two limits.

We have also analyzed the behavior of the second cumulant $\mathcal{S}_{2}$ in the presence of electron-electron interactions. We have demonstrated that Coulomb interaction decreases the Nyquist noise as one could

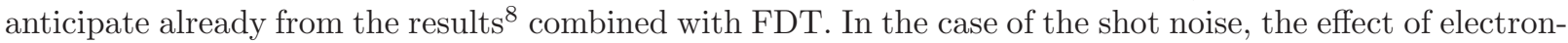
electron interactions turns out to be more complicated. The corresponding interaction correction was found negative for conductors with $\beta>2 \gamma$ and positive otherwise.

Finally, let us point out that our predictions can be experimentally tested in various types of coherent mesoscopic conductors, such as, e.g., break junctions, quantum point contacts or short diffusive metallic bridges. ${ }^{25,26}$ In all these systems both $\tau_{D}$ and $\tau_{R C}$ can be small enough in order to satisfy all the assumptions adopted here. For instance, in diffusive samples ${ }^{25,26}$ one finds $1 / \tau_{D}$ of order few Kelvins and, hence, the condition $\mathrm{eV}<1 / \tau_{D}$ is obeyed in a wide range of voltages $V<0.1 \div 0.5 \mathrm{mV}$. We also note that the work ${ }^{26}$ reports the experimental analysis of the frequency dispersion of shot noise ${ }^{1}$ which is important in the same frequency range as that of the third cumulant studied here.

This work is part of the Kompetenznetz "Funktionelle Nanostructuren" supported by the Landestiftung Baden-Württemberg gGmbH and of the STReP "Ultra-1D" supported by the EU.

\section{REFERENCES}

1. Ya.M. Blanter and M. Büttiker, Phys. Rep. 336, 1 (2000).

2. B. Reulet, J. Senzier, and D.E. Prober, Phys. Rev. Lett. 91, 196601 (2003).

3. L.S. Levitov, H.W. Lee, and G.B. Lesovik, J. of Math. Phys. 37, 4845 (1996).

4. H. Lee, L.S. Levitov, and A.Yu. Yakovets, Phys. Rev. B 51, 4079 (1995).

5. L.S. Levitov and M. Reznikov, cond-mat/0111057.

6. K.E. Nagaev, Phys. Rev. B 66, 075334 (2002).

7. D.B. Gutman and Yu. Gefen, Phys. Rev. B 68, 035302 (2003).

8. D.S. Golubev and A.D. Zaikin, Phys. Rev. Lett. 86, 4887 (2001).

9. A.V. Galaktionov, D.S. Golubev, and A.D. Zaikin, Phys. Rev. B 68, 085317 (2003).

10. A.V. Galaktionov, D.S. Golubev, and A.D. Zaikin, Phys. Rev. B 68, 235333 (2003).

11. M. Kindermann and Yu.V. Nazarov, Phys. Rev. Lett. 91, 136802 (2003).

12. D.A. Bagrets and Yu.V. Nazarov, cond-mat/0304339.

13. D.S. Golubev and A.D. Zaikin, Phys. Rev. B 59, 9195 (1999).

14. M. Kindermann and Yu.V. Nazarov, cond-mat/0303590.

15. G. Schön and A.D. Zaikin, Phys. Rev. B 40, 5231 (1989); A.D. Zaikin, Physica B 203, 255 (1994).

16. G. Schön and A.D. Zaikin, Phys. Rept. 198, 237 (1990).

17. Yu.V. Nazarov, Phys. Rev. Lett. 82, 1245 (1999).

18. A. Andreev and A. Kamenev, Phys. Rev. Lett. 85, 1294 (2000); L.S. Levitov, cond-mat/0210284.

19. K.A. Matveev, Phys. Rev. B 51, 1743 (1995).

20. G.B. Lesovik and N.M. Chtchelkatchev, cond-mat/0303024.

21. C.W.J. Beenakker, M. Kindermann, and Yu.V. Nazarov, Phys. Rev. Lett. 90, 176802 (2003).

22. A.J. Dahm et al., Phys. Rev. Lett. 22, 1416 (1969).

23. H. Lee and L.S. Levitov, Phys. Rev. B 53, 7383 (1996).

24. D.S. Golubev and A.D. Zaikin, Phys. Rev. B 69, 075318 (2004); cond-mat/0402306.

25. H. Weber, R. Häussler, H. von Lohneysen, and J. Kroha, Phys. Rev. B 63, 165426 (2001).

26. R.J. Schoelkopf et al., Phys. Rev. Lett. 78, 3370 (1997). 